\title{
Splenic artery aneurysm with the double-rupture phenomenon
}

\author{
Jung Ho Kim, Han Sol Chung, Jong Ha Kim, Sin Youl Park, Sam Beom Lee, \\ Byung Soo Do
}

Department of Emergency Medicine, Yeungnam University College of Medicine, Daegu, Korea

Splenic artery aneurysm is the third most common type of intra-abdominal aneurysm, with a prevalence rate of $0.01 \%$ to $10.4 \%$ in the general population. Splenic artery aneurysm is usually asymptomatic and is typically detected by chance and does not require surgical management; however, if rupture occurs, although rare, the patient's situation can become critical. We report our experience with a man who presented with left flank and left shoulder pain. His symptoms were caused by multiple hematomas confined to the spleen, but 2 days after admission, he developed delayed hemoperitoneum and required surgical management. We believe that his condition was due to delayed intraperitoneal bleeding called the double-rupture phenomenon; emergency physicians must consider this phenomenon when taking care of splenic artery aneurysm patients.

Keywords Splenic artery; Aneurysm; Double rupture

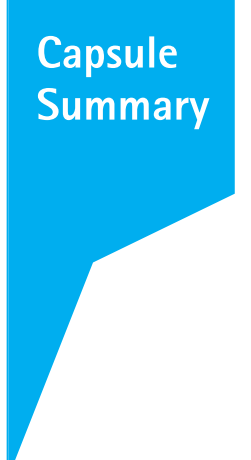

What is already known

Splenic artery aneurysm is rare and mostly asymptomatic but can lead to lifethreatening complications.

What is new in the current study

Some ruptured splenic artery aneurysms present with intraperitoneal bleeding 6 to 96 hours after the primary rupture. This time interval can provide a diagnostic window and a chance to save a patient's life.
Received: 7 February 2017

Revised: 28 February 2017

Accepted: 20 March 2017

Correspondence to: Sam Beom Lee Department of Emergency Medicine, Yeungnam University College of Medicine, 170 Hyeonchung-ro, Namgu, Daegu 42415, Korea E-mail: sblee@med.yu.ac.kr

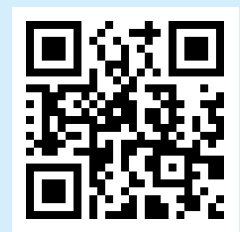

How to cite this article: Kim JH, Chung HS, Kim JH, Park SY, Lee SB, Do BS. Splenic artery aneurysm with the double-rupture phenomenon. Clin Exp Emerg Med 2017;4(2):113-116.

This is an Open Access article distributed under the terms of the Creative Commons Attribution Non-Commercial License (http:// creativecommons.org/licenses/by-nc/4.0/). 


\section{INTRODUCTION}

Abdominal pain is a common symptom resulting in emergency room visits. In the majority of cases, emergency physicians can find the cause of the pain, but occasionally, diagnosis can become very difficult because of an uncommon disease or an unusual course. Splenic artery aneurysm (SAA) is the third most common type of intra-abdominal aneurysm. Approximately $80 \%$ of SAAs are asymptomatic and are detected incidentally during diagnostic imaging performed for other indications. Approximately $20 \%$ of symptomatic patients show various symptoms, including abdominal pain, anorexia, nausea, and vomiting. ${ }^{1,2}$ Management of SAA depends on the symptoms, location and size of the aneurysm, and the risk of rupture; if needed, a surgical approach is sometimes required. The most serious complication of SAA is spontaneous rupture, which occurs in $2 \%$ to $10 \%$ of symptomatic patients. The mortality rate is approximately $25 \%$ of all symptomatic patients. With a ruptured SAA, delayed intraperitoneal bleeding occasionally occurs 6 to 96 hours later, with blood initially contained within the lesser omental sac. ${ }^{1-3}$ We report a patient who presented with acute left flank pain caused by multiple splenic hematomas without intraperitoneal bleeding and who developed hemoperitoneum with hemodynamic instability 72 hours after symptom onset.

\section{CASE REPORT}

A 51-year-old man visited the emergency department with sudden left flank and left shoulder pain that had developed 15 hours previously. Roughly 15 days before his visit, he had transiently experienced fever and upper respiratory symptoms, which subsided in a few days. Recently, he had been experiencing postprandial abdominal pain, even after a small amount of food. He had no previous medical history or recent traumatic event. He smoked a quarter of a pack per day for 30 years and drank alcohol socially.

His vital signs were stable, and he had left costo-vertebral angle tenderness, but neither abdominal tenderness nor peritoneal irritation on physical examination.

Initial laboratory results showed hemoglobin $14.2 \mathrm{~g} / \mathrm{dL}$, total bilirubin $2.66 \mathrm{mg} / \mathrm{dL}$, direct bilirubin $0.57 \mathrm{mg} / \mathrm{dL}$, aspartate transaminase $46 \mathrm{IU} / \mathrm{L}$, alanine transaminase $62 \mathrm{IU} / \mathrm{L}$, lactic dehydrogenase $653 \mathrm{IU} / \mathrm{L}$, and C-reactive protein $2.892 \mathrm{mg} / \mathrm{dL}$. As radiography showed no specific abnormal finding, we decided to perform an abdominal computed tomography (CT) scan. The initial CT scan revealed multiple hematomas confined to the spleen and a 4.5-cm aneurysm without hemoperitoneum (Fig. 1).

He was hospitalized in the general surgery department for conservative treatment. Two days after admission, he developed chest discomfort and his blood pressure became $80 / 60 \mathrm{mmHg}$. A follow-up abdominal CT scan was performed, which showed an enlarged spleen with multiple hematomas and newly developed hemoperitoneum around the spleen (Fig. 2).

He was sent to the operating room for splenectomy and removal of intraperitoneal blood. During the operation, hemoperitoneum due to a spleen laceration was identified. The gross specimen after splenectomy showed multiple, dark red hemorrhagic cysts, the largest one $(4 \times 4.5 \mathrm{~cm})$ located at the periphery of the spleen, with serosal rupture, which was diagnosed as an aneurysmal hematoma (Fig. 3). He was discharged on the seventh day after surgery without complications.
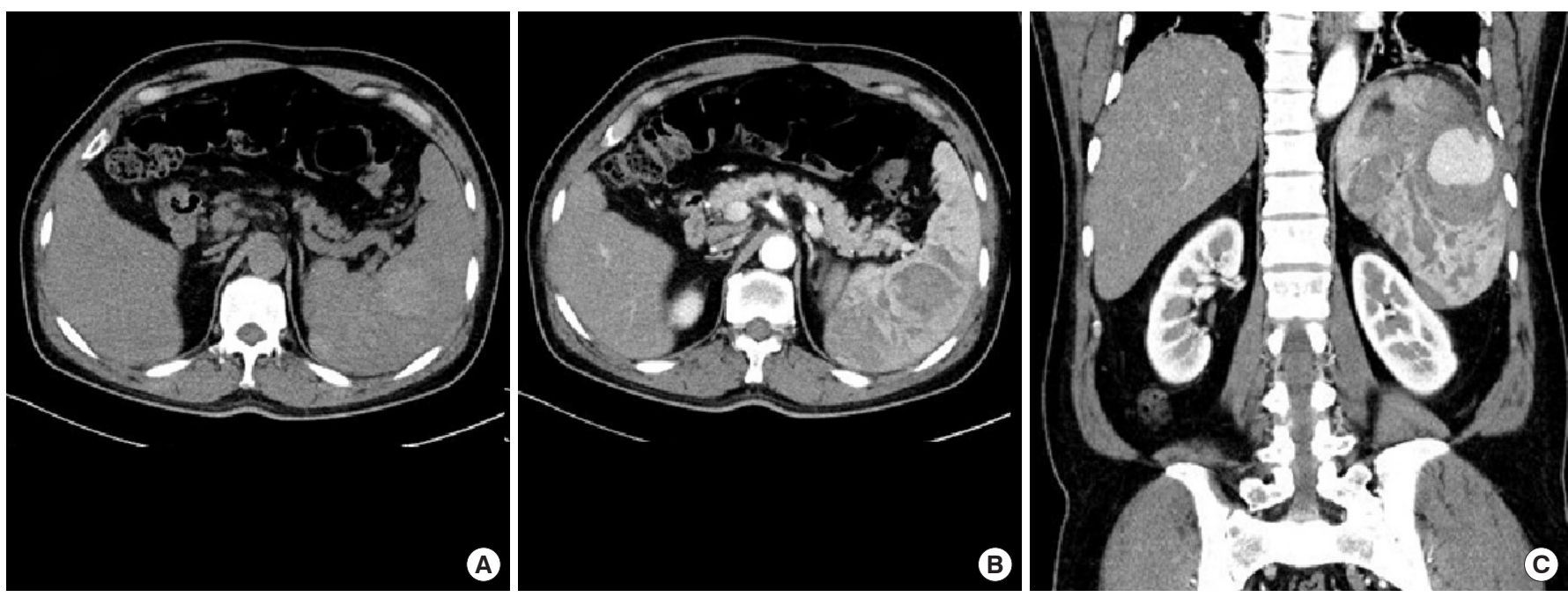

Fig. 1. Abdominal computed tomography scan shows multiple hematomas confined in the spleen and no hemoperitoneum around the spleen at the emergency room. (A) Non-enhanced scan, (B) enhanced scan, and (C) coronal view. 

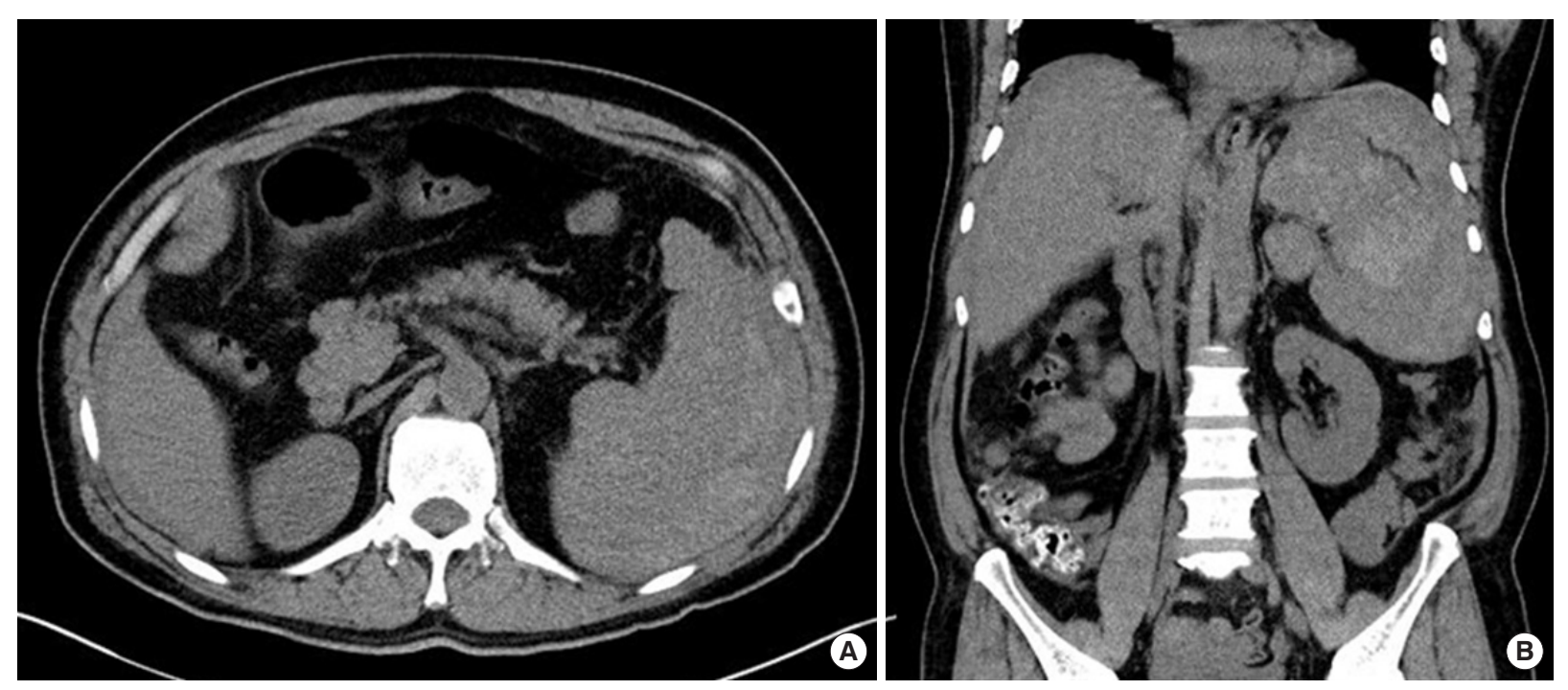

Fig. 2. Abdominal computed tomography scan shows multiple splenic hematomas and delayed hemoperitoneum around the spleen at 2 days after admission. (A) Non-enhanced scan and (B) non-enhanced coronal view.

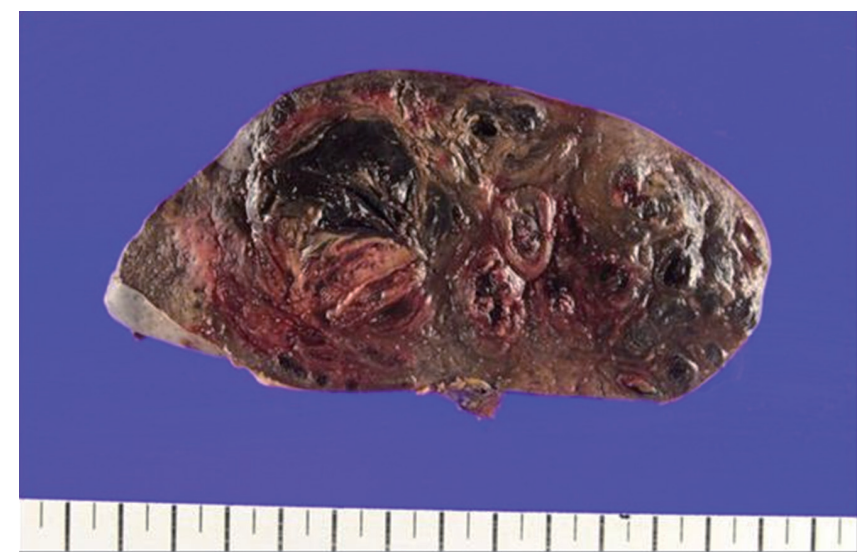

Fig. 3. Gross specimen after splenectomy showing the multiple, dark red hemorrhagic cysts, the largest one $(4 \times 4.5 \mathrm{~cm})$ located at the periphery of the spleen, with serosal rupture.

\section{DISCUSSION}

SAA is the third most common type of abdominal aneurysm, preceded only by aortic and iliac artery aneurysms, and is the most common splanchnic aneurysmal disease. SAA is defined as an abnormal dilatation of the splenic artery more than $1 \mathrm{~cm}$ in diameter. It was first described in 1770 during autopsy by Beaussier. ${ }^{1}$

The prevalence of SAA is $0.8 \%$ in unselective visceral angiograms and $0.1 \%$ to $10.4 \%$ in the general population, but the true prevalence remains unknown because most are asymptomatic. ${ }^{1,4}$ It is four times more common in female than male patients and most frequently encountered in women with multiple pregnancies. ${ }^{1,5}$ Risk factors include hormonal and local hemodynamic events in pregnancy, trauma, portal hypertension, Caroli syndrome, arterial degeneration, medial fibrodysplasia, and atherosclerosis. Its pathogenesis is still not fully understood. ${ }^{1.4,5}$

Approximately $80 \%$ to $95 \%$ of SAAs are asymptomatic and are mostly detected incidentally during diagnostic imaging performed for other indications. In symptomatic patients, vague abdominal pain is the most common clinical manifestation, especially in the epigastric or left upper quadrant area, and other symptoms such as nausea or vomiting can be seen. ${ }^{1,6}$

Despite being rare, SAAs can result in life-threatening complications, such as fistula formation into neighboring organs, splenic arteriovenous fistula formation, and spontaneous rupture. Spontaneous rupture is the most dangerous complication, with mortality rates of $25 \%$ to $75 \%$, because of intraperitoneal hemorrhage and hypovolemic shock. ${ }^{1,6}$ The high mortality is due to its asymptomatic nature before rupture, rapid aggravation after rupture, and frequent misdiagnosis. Factors such as pregnancy, expanding aneurysm, aneurysm over $2 \mathrm{~cm}$ in diameter, and portal hypertension with liver transplant or portacaval shunt are associated with an increased risk of rupture. ${ }^{2,7}$

If the SAA ruptures, patients present with sudden-onset epigastric or left upper quadrant abdominal pain, left shoulder tip pain called Kehr's sign, chest pain, and hemodynamic instability. Uncommonly, aneurysms may rupture initially into the lesser omental sac with transient tamponade; at this time, the patient remains stable, with minimal clinical symptoms. After 6 to 96 hours, the blood overflows into the peritoneal cavity through the foramen of Winslow, resulting in unexpected hemorrhagic shock and vascular collapse. This delayed blood loss is called the dou- 
ble-rupture phenomenon, described for the first time in 1930 by Bockerman. ${ }^{1}$ The initial phase where hemorrhage remains confined in the lesser omental sac can provide vital time for diagnosis and lifesaving treatment. ${ }^{1-4}$

In our case, the patient was, unusually, a middle-aged man with no risk factors for SAA, presenting with left flank and left shoulder pain. However, he presented with hemodynamic instability and new-onset hemoperitoneum 72 hours from symptom onset caused by a ruptured SAA. We supposed he experienced the double-rupture phenomenon, allowing time for diagnosis before progressing into a critical situation.

Contrast-enhanced CT scanning, ultrasonography, pulsed Doppler, magnetic resonance imaging, magnetic resonance angiography, and abdominal aortic arteriography are modalities that may be used for the diagnosis of an asymptomatic aneurysm. Generally, contrast-enhanced CT scanning is the primary method to detect the aneurysm and intraperitoneal hemorrhage; abdominal aortic angiography, however, still remains the most valuable method of choice to accurately localize the source of bleeding and assess collateral blood flow. ${ }^{3}$

Decisions regarding management are related to the risk of spontaneous rupture and every symptomatic patient should be considered urgent. ${ }^{1,6}$ A number of ways for managing SAAs have been reported. Traditional treatment is open surgical intervention, including aneurysm ligation with or without splenectomy and aneurysmal resection with revascularization. If the aneurysm is located in the hilar and intra-parenchymal area, splenectomy is inevitable. ${ }^{8}$ Treatment for rupture in a patient with hemodynamic instability is immediate open surgery, resection of the aneurysm, with or without splenectomy. ${ }^{9}$ In the present case, the aneurysm had an intra-parenchymal location and the patient presented with hemodynamic instability; therefore, a splenectomy was required. With the recent development of therapies, management options have expanded, including the use of laparoscopic surgery and nonsurgical endovascular management; however, these are suitable only for selected patients, and limited data about outcomes are available. ${ }^{7,10}$

SAA is rare and generally asymptomatic but, if ruptured, can result in a life-threatening condition. Rarely, a ruptured aneurysm can present with the double-rupture phenomenon, in which the aneurysm ruptures into the lesser omental sac primarily and blood overflows into the peritoneal cavity after 6 to 96 hours. The patient remains stable during the primary rupture, and rapid diagnosis and treatment during this time interval can be lifesaving and may improve outcomes. Therefore, emergency physicians should be reminded of this phenomenon when managing SAA patients, even if they are stable. This should always be considered if abrupt shock occurs. In addition, fluid resuscitation with appropriate and immediate shock management should be performed and surgical treatment should be considered.

\section{CONFLICT OF INTEREST}

No potential conflict of interest relevant to this article was reported.

\section{REFERENCES}

1. Al-Habbal $Y$, Christophi $C$, Muralidharan V. Aneurysms of the splenic artery: a review. Surgeon 2010;8:223-31.

2. Deshpande AA, Kulkarni VM, Rege S, Dalvi AN, Hardikar JV. Ruptured true aneurysm of the splenic artery: an unusual cause of haemoperitoneum. J Postgrad Med 2000;46:191-2.

3. Davis T, Minardi J, Knight J, Larrabee H, Schaefer G. Ruptured splenic artery aneurysm: rare cause of shock diagnosed with bedside ultrasound. West J Emerg Med 2015;16:762-5.

4. Oakley E, Ho JD, Johnson V, Vancamp J, Melson T, Hick JL. Splenic artery aneurysm: an important cause of hemoperitoneum and shock. J Emerg Med 2014;46:e65-7.

5. Liu CF, Kung CT, Liu BM, Ng SH, Huang CC, Ko SF. Splenic artery aneurysms encountered in the ED: 10 years' experience. Am J Emerg Med 2007;25:430-6.

6. Akbulut $\mathrm{S}, \mathrm{Otan}$ E. Management of giant splenic artery aneurysm: comprehensive literature review. Medicine (Baltimore) 2015;94:e1016.

7. Lakin RO, Bena JF, Sarac TP, et al. The contemporary management of splenic artery aneurysms. J Vasc Surg 2011;53:95864.

8. Pulli R, Dorigo W, Troisi N, Pratesi G, Innocenti AA, Pratesi C. Surgical treatment of visceral artery aneurysms: a 25-year experience. J Vasc Surg 2008;48:334-42.

9. Betal D, Khangura JS, Swan PJ, Mehmet V. Spontaneous ruptured splenic artery aneurysm: a case report. Cases J 2009;2: 7150.

10. Fankhauser GT, Stone WM, Naidu SG, et al. The minimally invasive management of visceral artery aneurysms and pseudoaneurysms. J Vasc Surg 2011;53:966-70. 